'Escuela de Medicina, Campus San Felipe, Universidad de Valparaíso. San Felipe, Chile. Escuela de Tecnología Médica, Campus San Felipe, Universidad de Valparaíso. San Felipe, Chile. ${ }^{3}$ Laboratorio de Bacteriología, Instituto de Salud Pública de Chile. Santiago, Chile. 4Laboratorio de Microbiología Clínica Río Blanco. Los Andes, Chile.

${ }^{5}$ Laboratorio de Bacteriología, Instituto Ramón y Cajal de Investigación Sanitaria. Madrid, España.

${ }^{6}$ Centro Interdisciplinario de Investigación en Salud Territorial del Valle de Aconcagua, Universidad de Valparaíso. San Felipe, Chile. aBioquímico. bPhD.

'Tecnólogo Médico. ¿Estudiante de Tecnología Médica. eEstudiante de Medicina.

Apoyo financiero: Dirección de Investigación Universidad de Valparaíso. Proyecto DIUV $N^{\circ} 71 / 2011$.

La ayuda recibida para la ejecución del proyecto a través de la dirección de Investigación Universidad de Valparaíso y la colaboración del ISP Chile al donar las cepas bacterianas para el estudio, no influenció en el diseño del estudio, en la recolección, análisis o interpretación de los datos, ni en la preparación, revisión o aprobación del manuscrito. Por tanto, no existe conflicto de interés con ninguna de las instituciones que apoyaron este proyecto.

Recibido el 6 de septiembre de 2018, aceptado el 18 de diciembre de 2018.

Correspondecia a: Carmen Aravena Molló Avda. Miraflores 2085 A. Anexo HOSCA. Casilla 296. San Felipe $\checkmark$ Región. carmen.aravena@uv.c

\section{Caracterización de cepas clínicas y ambientales de Salmonella enterica subsp. enterica serovar Heidelberg aisladas en Chile}

\author{
CARMEN ARAVENA ${ }^{1,6, a, b}$, BÁRBARA VALENCIA ${ }^{2, \mathrm{~d}}$, \\ ANDREA VILLEGAS ${ }^{2, \mathrm{~d}}$, MAURICIO ORTEGA ${ }^{1, \mathrm{e}}$, \\ ALDA FERNÁNDEZ R. ${ }^{3, \mathrm{c}}$, PAMELA ARAYA R. ${ }^{3, \mathrm{a}}$, \\ ANÍBAL SAAVEDRA ${ }^{2,4, \mathrm{c}}$, ROSA DEL CAMPO ${ }^{5, \mathrm{~b}}$
}

\section{Characterization of Salmonella Heidelberg strains isolated in Chile}

\begin{abstract}
Background: Salmonella Heidelberg (S. Heidelberg) causes gastroenteritis and sometimes bacteremia and endocarditis. In other countries, this serovar has multidrug resistance including extended-spectrum $\beta$-lactamases (ESBLs) and AmpC $\beta$-lactamases $(A m p C)$, associated with the bla ${ }_{C M Y-2}$ gene. In Chile, an outbreak by S. Heidelberg occurred in 2011, the phenotypic and genetic characteristics of Chilean strains are unknown. Aim: To determine the antimicrobial susceptibility, presence of plasmids and virulence factor genes in S. Heidelberg strains isolated in Chile over the period 2006-2011. Material and Methods: In sixty-one S. Heidelberg clinical and environmental strains collected by the Public Health Institute in Chile during 2006-2011, antimicrobial susceptibility, plasmids and virulence factor genes (invA, sifA, pefA, agfA, lpfA and, stkD) were studied. Results: S. Heidelberg had a high susceptibility to sulfamethoxazole-trimethoprim, gentamicin, ceftriaxone, ceftiofur, chloramphenicol, amoxicillin-clavulanic acid and ampicillin. However, $52 \%$ had decreased susceptibility to ciprofloxacin and 33\% resistance to tetracycline. ESBLs were detected in three strains isolated from blood cultures, environment and human feces. The latter strain was positive for AmpC and bla $a_{C M Y-2}$ gene. Fifty three of 61 strains showed one to seven plasmids of 0.8 to approximately $30 \mathrm{~kb}$. Most plasmids were small with sizes between 0.8 and $2 \mathrm{~kb}$. All isolates were positive for all genes except pefA. Conclusions: S. Heidelberg isolated from Chilean samples was susceptible to first-line antimicrobials, except tetracycline and ciprofloxacin. The emergence of strains with ESBLs and AmpC should be a warning. The strains were homogeneous for virulence genes, but heterogeneous in their plasmids.
\end{abstract}

(Rev Med Chile 2019; 147: 24-33)

Key words: Drug Resistance; Gastroenteritis; Plasmids; Salmonella enterica.
S almonella enterica subsp. enterica serovar Heidelberg, que por convención y simplicidad nombraremos como $S$. Heidelberg, es uno de los serovares de Salmonella más frecuentemente aislados en casos de gastroenteritis en Canadá y Estados Unidos de Norteamérica ${ }^{1,2}$. Carnes de aves de corral y huevos la principal fuente de transmisión a humanos ${ }^{3,4}$. S. Heidelberg ha mostrado ser muy invasiva causando septicemia y endocarditis $^{5-7}$. En Brasil, África y en Norteamérica 
se han descrito cepas de $S$. Heidelberg multirresistentes a los antimicrobianos provenientes de carnes de ave, cerdos y huevos ${ }^{1,4,8,9}$, lo que se atribuye al uso de ceftiofur y también de florfenicol en la producción animal ${ }^{4,5,10}$. Particularmente, la resistencia a ceftiofur, una cefalosporina de tercera generación, se asocia a la resistencia múltiple a drogas y a la presencia del gen $b l a_{\mathrm{CMY}-2}$ que codificaría para una $\beta$-lactamasa plasmidial tipo AmpC, presente en enterobacterias y muy prevalente en Escherichia coli y Salmonella $a^{4,9,11-14}$.

En $S$. Heidelberg, variaciones en la capacidad de invasión y colonización de las cepas se han asociado a diferencias en la presencia de genes de virulencia (invA, agfA y $l p f A$ ) y en el patrón de electroforesis en gel de campo pulsado ${ }^{6,15}$.

En Chile, Salmonella Enteritidis es el serovar causante de gastroenteritis más frecuente en los últimos 20 años ${ }^{16,17}$; sin embargo, se observó un incremento de $S$. Heidelberg en 2011 respecto a los años 2006-2010 (Fernández A et al. 2011, X Jornadas Científicas, Instituto de Salud Pública de Chile). Esto se puede atribuir al surgimiento de nuevas variantes genéticas con mayor capacidad patógena, aunque actualmente se desconocen las características fenotípicas y genotípicas de las cepas de $S$. Heidelberg que circulan en nuestro país. Por ello, el objetivo de este estudio fue caracterizar la susceptibilidad antimicrobiana, la presencia de plásmidos, genes de resistencia y de virulencia de cepas de $S$. Heidelberg aisladas en Chile en el período mencionado.

\section{Material y Métodos}

\section{Cepas bacterianas}

Sesenta y un aislamientos (32 clínicos y 29 ambientales o no humanos) de $S$. Heidelberg de diferentes partes de Chile y remitidos al Instituto de Salud Pública de Chile (ISP Chile) entre los años 2006 y 2011, se seleccionaron aleatoriamente entre 9 y 10 aislamientos por año, considerando ambos orígenes (Tabla 1). S. Heidelberg se identificó por técnicas microbiológicas convencionales y por serología según métodos estándar en ISP Chile ${ }^{18}$. Para diferentes pruebas se usaron cepas controles: $E$. coli ATCC 25922 y Salmonella Typhimurium LT2.

\section{Susceptibilidad antimicrobiana}

Se realizó por método de difusión en agar con sensidiscos ${ }^{19}$. Se utilizó agar Mueller Hinton (Val- tek Diagnostics, Chile) y sensidiscos de: ceftiofur $(30 \mu \mathrm{g})$, sulfametoxazol-trimetoprim $(25 \mu \mathrm{g})$ y cloranfenicol $(30 \mu \mathrm{g})$ (Oxoid, Reino Unido). Para amoxicilina-ácido clavulánico, tetraciclina, gentamicina, ciprofloxacino, ampicilina y ceftriaxona se determinó la concentración inhibitoria mínima (CIM) usando M.I.C. Evaluator ${ }^{\mathrm{TM}}$ (Oxoid, Reino Unido). Ambos métodos se realizaron en condiciones estándar y por duplicado para cada aislamiento, la interpretación fue según los criterios del Clinical and Laboratory Standards Institute ${ }^{20}$. BLEE y AmpC se determinaron mediante test de inhibición con sensidiscos utilizando ceftazidima $(30 \mu \mathrm{g})$, ceftazidima-clavulanato $(30 / 10 \mu \mathrm{g})$ y cefotaxima $(30 \mu \mathrm{g})$ y cefotaxima-clavulanato $(30 / 10$ $\mu \mathrm{g})$ utilizando los criterios para K. pneumoniae ${ }^{19}$.

\section{Extracción de plásmidos}

Una o 2 colonias de $S$. Heidelberg crecidas en agar Salmonella-Shigella (HiMedia, India) se cultivaron en $10 \mathrm{ml}$ de caldo Luria Bertani (Omega Bio-Tek, USA) a $37^{\circ} \mathrm{C}$ por $18 \mathrm{~h}$, para obtener luego el ADN plasmídico con kit de extracción E.Z.N.A. Plasmid Mini Kit II (Omega Bio-Tek, USA). El ADN se cuantificó por fotometría y 0,5 $\mu \mathrm{g}$ de ADN plasmidial se mezclaron con tampón de carga ADN 6X (Thermo Scientific) incubando a $100{ }^{\circ} \mathrm{C}$ por $3 \mathrm{~min}$ para evitar la formación concatámeros. Los plásmidos fueron separados por electroforesis en gel de agarosa al 0,7\%, según Takahashi y Nagano, $1984^{21}$. El ADN teñido con Gel Red (Biotium, USA) fue visualizado en transiluminador UV. El tamaño de cada plásmido se determinó por comparación con fragmentos patrones de fago Lambda DNA/HindIII y 100 bp DNA Ladder (Thermo Ficher Scientific, Inc.).

\section{Extracción de ADN bacteriano}

Se realizó según Fadl et al., 1995²2. El ADN bacteriano se cuantificó y visualizó en las mismas condiciones que los plásmidos.

\section{Detección de genes}

Los genes agfA, lpfA, sifA, stkD, pefA, invA y el gen $b l a_{\mathrm{CMY}-2}$ fueron detectados por reacción en cadena de la polimerasa (RCP) de punto final, según condiciones indicadas en la Tabla 2. La secuencia nucleotídica parcial de los genes se obtuvo desde el producto de RCP usando como templado ADN de la cepa SH06. La secuenciación se realizó en secuenciador automático ABI PRISM 
Tabla 1. Cepas de S. Heidelberg estudiadas, código de identificación de cada cepa, año de recolección y origen $(n=61)$

\begin{tabular}{|c|c|c|c|c|c|}
\hline Cepa & Año & Origen & Cepa & Año & Origen \\
\hline $\mathrm{SHO} 1$ & 2006 & Clínico & $\mathrm{SH} 32$ & 2009 & Pavo \\
\hline $\mathrm{SHO2}$ & 2006 & Clínico & $\mathrm{SH} 33$ & 2009 & Clínico \\
\hline $\mathrm{SHO3}$ & 2006 & Animal n/e & $\mathrm{SH} 34$ & 2009 & Superficie n/e \\
\hline $\mathrm{SHO} 4$ & 2006 & Clínico & SH35 & 2010 & Pollo \\
\hline SH05 & 2006 & No humano n/e & SH36 & 2010 & Cerdo \\
\hline $\mathrm{SHO6}$ & 2006 & Clínico & SH37 & 2010 & Clínico \\
\hline $\mathrm{SHO7}$ & 2006 & Pavo crudo & $\mathrm{SH} 38$ & 2010 & Aves n/e \\
\hline $\mathrm{SHO8}$ & 2006 & Clínico & SH39 & 2010 & Aves n/e \\
\hline $\mathrm{SH} 09$ & 2006 & Agua cloacas & $\mathrm{SH} 40$ & 2010 & Aves n/e \\
\hline SH10 & 2007 & Clínico & $\mathrm{SH} 41$ & 2010 & Pollo \\
\hline SH11 & 2007 & Clínico & $\mathrm{SH} 42$ & 2010 & Pollo \\
\hline $\mathrm{SH} 12$ & 2007 & Clínico & $\mathrm{SH} 43$ & 2010 & Clínico \\
\hline SH13 & 2007 & Pavo crudo & $\mathrm{SH} 44$ & 2010 & Carne de pavo \\
\hline $\mathrm{SH} 14$ & 2007 & Ensalada surtida & $\mathrm{SH} 45$ & 2010 & Clínico \\
\hline SH15 & 2007 & Clínico & $\mathrm{SH} 46$ & 2010 & Clínico \\
\hline SH16 & 2007 & No humano n/e & $\mathrm{SH} 47$ & 2010 & Clínico \\
\hline $\mathrm{SH} 17$ & 2007 & Clínico & $\mathrm{SH} 48$ & 2010 & Pollo \\
\hline SH18 & 2008 & Clínico & SH49 & 2010 & Ave \\
\hline $\mathrm{SH} 19$ & 2008 & Clínico & SH50 & 2010 & Pavo \\
\hline $\mathrm{SH} 20$ & 2008 & Clínico & SH51 & 2010 & Clínico \\
\hline $\mathrm{SH} 21$ & 2008 & Carne pollo & SH52 & 2011 & Clínico \\
\hline $\mathrm{SH} 22$ & 2008 & Jamón de cerdo & SH53 & 2011 & No humano n/e \\
\hline $\mathrm{SH} 23$ & 2008 & Pollo & SH54 & 2011 & Clínico \\
\hline $\mathrm{SH} 24$ & 2008 & Alimento n/e & SH55 & 2011 & Clínico \\
\hline $\mathrm{SH} 25$ & 2008 & Clínico & SH56 & 2011 & Clínico \\
\hline $\mathrm{SH} 26$ & 2009 & Pavo cocido & SH57 & 2011 & Clínico \\
\hline $\mathrm{SH} 27$ & 2009 & Clínico & SH58 & 2011 & Clínico \\
\hline $\mathrm{SH} 28$ & 2009 & Clínico & SH59 & 2011 & Clínico \\
\hline $\mathrm{SH} 29$ & 2009 & Clínico & SH60 & 2011 & Pollo \\
\hline $\mathrm{SH} 30$ & 2009 & Ciegos de rata & SH61 & 2011 & Ave \\
\hline SH 31 & 2009 & Clínico & & & \\
\hline
\end{tabular}

n/e= no especificado; clínico = cepas obtenidas desde pacientes humanos.

310 Genetic Analyzer (Applied Biosystem) según Sanger et al., $1977^{28}$. Las secuencias fueron ingresadas en GenBank : bla $a_{\mathrm{CMY}-2}$ (KP340125), invA (KX267824), agfA (KY564364), sifA (KY564365) y stkD (KY564366).

\section{Conjugación bacteriana}

Para la conjugación se usaron como dadoras las cepas de $S$. Heidelberg resistentes a ceftriaxona y a ceftiofur y como cepa receptora una E. coli K-12 (Bio-Rad, USA) resistente a rifampicina 
Tabla 2. Partidores utilizados para la detección de genes de virulencia en S. Heidelberg. Se indica el tamaño de la región amplificada, la temperatura utilizada en la etapa de annealing y la publicación de donde se obtuvo la secuencia de los partidores

\begin{tabular}{|c|c|c|c|c|}
\hline Gen & Partidores & $\begin{array}{c}\text { Tamaño } \\
\text { pb }\end{array}$ & $\mathbf{T}^{\circ}$ annaeling & Referencia \\
\hline $\operatorname{agfA}$ & $\begin{array}{l}\text { F: tccacaatggggcggcggcg } \\
\text { R: cctgacgcaccattacgctg }\end{array}$ & 350 & $54^{\circ} \mathrm{C}$ & (23) \\
\hline bla ${ }_{\text {CMY-2 }}$ & $\begin{array}{l}\text { F: gacagcctctttctccaca } \\
\text { R: tggaacgaaggctacgta }\end{array}$ & 1.000 & $50^{\circ} \mathrm{C}$ & (24) \\
\hline IpfA & $\begin{array}{l}\text { F: ctttcgctgctgaatctggt } \\
\text { R: cagtgttaacagaaaccagt }\end{array}$ & 250 & $54^{\circ} \mathrm{C}$ & (25) \\
\hline pefA & $\begin{array}{l}\text { F: ttccattattgcactgggtg } \\
\text { R: aagccactgcgaaagatgcc }\end{array}$ & 496 & $62^{\circ} \mathrm{C}$ & (26) \\
\hline sifA & $\begin{array}{l}\text { F: tttgccgaacgcgccсcсacacg } \\
\text { R: gttgccttttcttgcgctttccacccatct }\end{array}$ & 449 & $66,5^{\circ} \mathrm{C}$ & (27) \\
\hline invA & $\begin{array}{l}\text { F: cgatagcctggcggtgggtttt } \\
\text { R: gcggggatctgggcgacaag }\end{array}$ & 411 & $62^{\circ} \mathrm{C}$ & Diseño propio \\
\hline$s t k D$ & $\begin{array}{l}\text { F: cccctgtggtagccgaattt } \\
\text { R: gcttgccgctaactccacta }\end{array}$ & 467 & $60^{\circ} \mathrm{C}$ & Diseño propio \\
\hline
\end{tabular}

$\mathrm{F}=$ forward; $\mathrm{R}=$ reverse; agfA $=$ gen de operón fimbria tipo $1 ;$ bla ${ }_{\mathrm{CMY}-2}=$ gen de $\beta$-lactamasa tipo AmpC; lpfA = gen de fimbria largo polar; pefA = gen adhesina de fimbria codificado en plásmido; sifA = gen de factor que favorece la multiplicación bacteriana en macrófago; invA = gen de factor de invasión epitelial; stkD = gen fimbrial putativo; $p b=$ pares de bases; $\mathrm{T}^{\circ}=$ temperatura; ${ }^{\circ} \mathrm{C}=$ grados Celsius.

(100 $\mu \mathrm{g} / \mathrm{mL})$, según protocolo de Phornphisutthimas et al. $2007^{29}$.

\section{Análisis estadístico}

Se agruparon las cepas de $S$. Heidelberg según la presencia de plásmidos de tamaño similar por análisis de clúster de tipo jerárquico, con software estadístico STATA 11 (StataCorp) para Windows (Microsoft Corporation).

\section{Electroforesis en gel de campo pulsado (EGCP)}

Se realizó EGCP a las cepas de $S$. Heidelberg BLEE positivas, según el protocolo de CDC Pulse Net (http.//www.cdc.gov/pulsenet/pathogens/ index.html, último acceso el 9 de julio de 2018) usando la enzima XbaI.

\section{Resultados}

\section{Susceptibilidad a los antimicrobianos}

Los aislamientos de $S$. Heidelberg clínicos y ambientales mostraron una susceptibilidad mayor al $90 \%$ en los antimicrobianos probados excepto para tetraciclina y ciprofloxacino (Tabla 3). Cepas de todos los años mostraron resistencia a tetraciclina con mayor proporción en cepas clínicas que en ambientales. Con ciprofloxacino, $S$. Heidelberg no mostró resistencia, pero $52,5 \%$ de las cepas tuvo susceptibilidad intermedia (CIM entre 0,12-0,5 $\mu \mathrm{g} / \mathrm{ml}$ ), disminuyendo la susceptibilidad a este antimicrobiano. De todos los aislamientos estudiados, 11 (18\%) fueron susceptibles a todos los antimicrobianos probados y $18(29,5 \%)$ mostraron resistencia a 1 o más antimicrobianos (12 clínicas y 6 ambientales), el resto fueron cepas con susceptibilidad intermedia a ciprofloxacino (resultados no se muestran). Dos cepas de $S$. Heidelberg del año 2006, una ambiental (SH05) y otra de deposiciones (SH06), más un aislado de hemocultivo (SH 52) año 2011, mostraron resistencia simultánea a ceftiofur, ampicilina, ceftriaxona y a amoxicilina-ácido clavulánico. Todas ellas fueron positivas para BLEE, aunque solo la cepa SH06 fue positiva para AmpC y para el gen bla $_{\text {CMY-2, }}$, además, esta cepa transfirió la resistencia a amoxicilina-ácido clavulánico a $E$. coli $\mathrm{K}-12$ por conjugación de un plásmido de cerca de $30 \mathrm{~kb}$, 
Tabla 3. Susceptibilidad antimicrobiana de $S$. Heidelberg. Se muestra el porcentaje de la susceptibilidad total de todas las cepas $(n=61)$ y la susceptibilidad según el origen de las cepas, aislamientos clínicos $(n=32)$ y ambientales $(n=29)$

\begin{tabular}{|c|c|c|c|c|c|}
\hline Antimicrobiano & Origen & $\% \mathbf{S}$ & $\%$ I & $\% \mathbf{R}$ & $\%$ S total \\
\hline \multirow[t]{2}{*}{ Ampicilina } & Clínico & 90,6 & 0 & 9,4 & \multirow[t]{2}{*}{91,8} \\
\hline & Ambiental & 93,1 & 0 & 6,9 & \\
\hline \multirow[t]{2}{*}{ Amoxicilina/ácido clavulánico } & Clínico & 93,8 & 0 & 6,3 & \multirow{2}{*}{93,4} \\
\hline & Ambiental & 93,1 & 0 & 6,9 & \\
\hline \multirow[t]{2}{*}{ Ceftriaxona } & Clínico & 93,8 & 0 & 6,3 & \multirow{2}{*}{95,1} \\
\hline & Ambiental & 96,6 & 0 & 3,4 & \\
\hline \multirow[t]{2}{*}{ Ceftiofur } & Clínico & 93,8 & 0 & 6,3 & \multirow{2}{*}{95,1} \\
\hline & Ambiental & 96,6 & 0 & 3,4 & \\
\hline \multirow[t]{2}{*}{ Gentamicina } & Clínico & 100 & 0 & 0 & \multirow{2}{*}{100} \\
\hline & Ambiental & 100 & 0 & 0 & \\
\hline \multirow[t]{2}{*}{ Tetraciclina } & Clínico & 50,0 & 18,8 & 31,3 & \multirow{2}{*}{50,8} \\
\hline & Ambiental & 51,7 & 34,5 & 13,8 & \\
\hline \multirow[t]{2}{*}{ Cloranfenicol } & Clínico & 96,9 & 3,1 & 0 & \multirow{2}{*}{95,1} \\
\hline & Ambiental & 93,1 & 6,9 & 0 & \\
\hline \multirow[t]{2}{*}{ Ciprofloxacino } & Clínico & 71,9 & 28,1 & 0 & \multirow{2}{*}{47,5} \\
\hline & Ambiental & 20,7 & 79,3 & 0 & \\
\hline \multirow[t]{2}{*}{ Sulfametoxazol-Trimetroprim } & Clínico & 100 & 0 & 0 & \multirow{2}{*}{100} \\
\hline & Ambiental & 100 & 0 & 0 & \\
\hline
\end{tabular}

$\%$ = porcetaje; $\mathrm{S}=$ susceptibilidad $; \mathrm{I}=$ Susceptibilidad intermedia; $\mathrm{R}=$ resistencia y $\mathrm{S}$ total = como susceptibilidad de todas las cepas.

como el detectado en la cepa dadora. El análisis por campo pulsado de las cepas BLEE positivas mostró que son clones estrechamente relacionados (los resultados no se muestran).

\section{Plásmidos en S. Heidelberg}

Cincuenta y tres cepas (87\%) mostraron desde uno a siete plásmidos, con tamaños desde 0,8 hasta cerca de $30 \mathrm{~kb}$, el resto, 7 aislamientos, no mostraron plásmidos. El análisis de clúster de los plásmidos de $S$. Heidelberg mostró que hay perfiles que se repiten en más de un aislado (grupos comunes), pero, además, hay una gran variabilidad respecto a los plásmidos presentes en cada cepa. Se obtuvieron once grupos comunes $(\mathrm{C} 1-\mathrm{C} 11)$ y 16 únicos (S1-S16) (Tabla 4). El grupo C1, con cepas mayoritariamente clínicas que mostraron dos pequeños plásmidos, y $\mathrm{C} 2$ correspondería a cepas en donde no se detectaron plásmidos. En C3, las cepas tenían tres plásmidos, un plásmido adicional de 1,4 $\mathrm{kb}$ respecto al grupo $\mathrm{C} 1$ compuesto por cepas de años anteriores. Similar observación al comparar C3 y C5 donde el plásmido adicional es de $1 \mathrm{~kb}$. En general, el número de plásmidos aumentó a medida que el año de recolección era más reciente, de cero a 2 plásmidos por cepa entre 2006-2007 y de 2-5 plásmidos entre el 2010-2011 (Tabla 4). La cepa SH35, con susceptibilidad intermedia a tetraciclina y a ciprofloxacino, mostró 7 plásmidos entre 0,8 y $4 \mathrm{~kb}$ (Tabla 4, S16). Este estudio evidenció que plásmidos de menos de $2 \mathrm{~kb}$ son frecuentes en $S$. Heidelberg y solo ocho cepas mostraron un plásmido de $30 \mathrm{~kb}$ que estaría como único plásmido o junto a otros pequeños plásmidos.

\section{Presencia de genes de virulencia}

Todas las cepas $S$. Heidelberg fueron positivas para los genes invA, sifA, stkD, agfA y $l p f A$, mientras que pefA no fue detectado en ningún aislamiento. 
Tabla 4. Agrupamiento de cepas de $S$. Heidelberg según presencia de plásmidos.

Para cada grupo se indica el tamaño de los plásmidos presentes o ausencia de ellos, el número de cepas que muestran la característica, el año y su origen $(n=61)$

\begin{tabular}{|c|c|c|c|}
\hline Grupo & Tamaño plásmidos (kb) & n de cepas & Año y origen de las cepas \\
\hline $\mathrm{C} 1$ & 0,8 y 1,3 & 9 & $\begin{array}{l}2006 \text { (1 clin.), } 2007 \text { (3 clin.), } 2008 \text { (2 amb. y } 1 \text { clin.), } \\
2009 \text { (1 clin.), } 2010 \text { (1 clin.) }\end{array}$ \\
\hline $\mathrm{C} 2$ & Sin plásmidos & 7 & 2006-2008 (2 ambientales y 5 clínicas) \\
\hline C3 & $0,8,1,3$ y 1,4 & 6 & 2010 (4 amb. y 1 clin.), 2011 (1 clin.) \\
\hline $\mathrm{C} 4$ & 1,7 & 5 & Todas ambientales: 2006 (1), 2007 (2), 2009 (1), 2010 (1). \\
\hline C5 & $0,8,1,1,3$ y 1,4 & 4 & 2010 (1 ambiental) y 2011 (2 clin. y 1 amb.) \\
\hline C6 & $1,7 y \approx 30$ & 4 & Todas ambientales 2007 (1), 2009 (3) \\
\hline $\mathrm{C7}$ & $0,8,1,3$ y 2,3 & 2 & 2008 y 2009 origen clínico \\
\hline $\mathrm{C} 8$ & $0,8,1$ y 1,3 & 2 & 2010 clínica \\
\hline C9 & 0,8 & 2 & 2007 y 2009 clínicas \\
\hline C10 & $0,8,1,3$ y 2 & 2 & 2009 clínica \\
\hline C11 & $0,8,1,3,1,4,1,7$ y 2 & 2 & 2010 (ambiental) y 2011 (clínica) \\
\hline S1 & 0,8 y 1,4 & 1 & 2010 clínica \\
\hline $\mathrm{S} 2$ & $0,8,1,3,1,7$ y 2 & 1 & 2010 ambiental \\
\hline S3 & $0,8,1,0,1,3$ y 2 & 1 & 2008 ambiental \\
\hline S4 & $1,3,1,4$ y 2,0 & 1 & 2010 clínica \\
\hline S5 & $0,8,0,9,1,3,1,4$ y 1,7 & 1 & 2010 ambiental \\
\hline S6 & $\approx 30$ & 1 & 2006 clínica \\
\hline S7 & $1,4 y \approx 30$ & 1 & 2006 ambiental \\
\hline S8 & 4 y 1,4 & 1 & 2010 ambiental \\
\hline s9 & 2,3 y 3,3 & 1 & 2008 clínica \\
\hline $\mathrm{S} 10$ & 1,3 y 2,2 & 1 & 2006 ambiental \\
\hline S 11 & $1,3,1,7,2,5$ y 30 & 1 & 2011 clínica \\
\hline $\mathrm{S} 12$ & $1,2,1,7,3,3$, y 30 & 1 & 2011 ambiental \\
\hline $\mathrm{S} 13$ & $0,8,1$ y 2 & 1 & 2011 clínica \\
\hline S14 & 1 y 2 & 1 & 2011 ambiental \\
\hline S15 & 0,8 y 1,7 & 1 & 2011 clínica \\
\hline S16 & $0,8,1,1,3,1,4,1,7,2,5$ y 4 & 1 & 2010 ambiental \\
\hline
\end{tabular}

$\mathrm{C}=$ grupo común con más de una cepa que comparten una característica; $\mathrm{S}=$ single o único que indica que solo una cepa tuvo la característica; $\mathrm{kb}=$ unidad de tamaño de los plásmidos en kilo pares de bases; clin. = clínica y amb. = ambiental, refiriéndose a cepas de procedencia no humano cuyo origen se especificó en la Tabla 1.

\section{Discusión}

Es importante estudiar las bacterias patógenas a nivel local para monitorear la aparición de clones virulentos y caracterizar las cepas circulantes ${ }^{30}$, además, es conocido que la susceptibilidad antimicrobiana de Salmonella enterica varía según su serovar, lugar geográfico, año de colección y origen $^{4}$. En $S$. Heidelberg se ha reportado alta resistencia antimicrobiana en aislados de carnes de pavo, pollo y cerdo en Estados Unidos de Norteamérica, $66 \%$ de los aislados fue resistente a un antimicrobiano y $16 \%$ resistente hasta a cinco antimicrobianos, y en Turquía, $41 \%$ fue resistente a uno o más antimicrobianos ${ }^{4,31}$. Comparado con nuestro estudio en cepas ambientales $(75 \%$ de 
procedencia animal), la resistencia a uno o más antimicrobianos fue baja $(20,7 \%)$ e inclusive menor que la reportada en países vecinos como Argentina (27\%) y Brasil $(29 \%)^{6,32}$. Sin embargo, cepas clínicas mostraron un porcentaje superior $(37,5 \%)$, atribuible principalmente a la resistencia a tetraciclina. En las cepas chilenas de $S$. Heidelberg, la baja susceptibilidad a tetraciclina se debió a la resistencia y susceptibilidad intermedia, lo que concuerda con otros estudios en S. enterica de diferentes orígenes aisladas en Chile (33, Prat S, et al., 2011, X Jornadas Científicas, ISP Chile). Estudios en Argentina de aislamientos animales de $S$. enterica y $S$. Heidelberg de origen animal y clínico mostraron resultados similares para tetraciclina ${ }^{4,32,34}$.

Más de la mitad de las cepas de $S$. Heidelberg del presente estudio mostraron susceptibilidad disminuida o intermedia a ciprofloxacino, lo que se ha descrito previamente en S. enterica ${ }^{35}$. El fenotipo resistente a ciprofloxacino se atribuye a mutaciones en el gen gyrA en los codones $83 \mathrm{u}$ 87 , sumado a la sobreexpresión de los genes marA y soxS, reguladores de bombas de eflujo ${ }^{35,36}$. Los resultados sugieren que las cepas chilenas aún no presentan estas características, sin embargo, el uso indiscriminado de estos antimicrobianos podría favorecer la aparición de resistencia a las fluoroquinolonas.

La resistencia bacteriana se atribuye al uso $\mathrm{y}$ abuso de los antimicrobianos en humanos y animales en forma terapéutica, subterapéutica, profilaxis y como promotor del crecimiento ${ }^{37,38}$. Estudios de brote por $S$. Heidelberg atribuidos al consumo de carnes de aves de corral en Estados Unidos de Norteamérica, muestran multirresistencia, con un patrón de resistencia a gentamicina, ampicilina, tetraciclina y estreptomicina ${ }^{2}$ y otro patrón con combinaciones de resistencia a gentamicina, ampicilina, tetraciclina, cloranfenicol, kanamicina, estreptomicina y sulfametoxazol-trimetropin en 35\% de los aislamientos clínicos ${ }^{39}$. En $S$. Heidelberg de origen chileno, solo una cepa de deposiciones (SH06) mostró multirresistencia a ampicilina, ceftriaxona, amoxicilina-ácido clavulánico, ceftiofur y tetraciclina, además, todas las cepas estudiadas por nosotros fueron susceptibles a gentamicina y a sulfametoxazol-trimetropin y no resistentes a cloranfenicol.

En Salmonella enterica subsp. enterica serovar Thyphimurium y en los serovares Heidelberg y
Newport la resistencia a ceftiofur se relaciona con la resistencia a ceftriaxona, que puede deberse a una $\beta$-lactamasa del tipo AmpC plasmidial que presenta el gen $b l a_{\mathrm{CMY}-2}$ y que se puede transferir por conjugación ${ }^{13,38}$. En nuestro estudio, cepas resistentes a ceftriaxona y a ceftiofur fueron BLEE positivas, pero solo la cepa SH06 (2006) fue positiva para AmpC y bla ${ }_{\mathrm{CMY}-2}$. S. Heidelberg con estas características se han reportado en distintas partes del mundo ${ }^{4,11,14,33,40}$. En nuestro estudio, el análisis por EGCP de las cepas BLEE positivas mostró que existe entre ellas una estrecha relación clonal, es decir, provienen de un mismo clon de $S$. Heidelberg y, además, este clon está circulando en el ambiente (cepa SH05), causando infecciones intestinales (cepa SH06) e infecciones invasivas (cepa SH52), al menos el período de este estudio. Aunque cabe mencionar que a través del análisis de plásmidos y el antibiotipo fue posible establecer diferencias entre estas cepas.

Los resultados obtenidos por nosotros coinciden con reportes de plásmidos en $S$. Heidelberg de tamaños menores a 2 y hasta $7 \mathrm{~kb}$ y de $34 \mathrm{~kb}^{31,34,40}$. También, un plásmido de $3,3 \mathrm{~kb}$ en $S$. Heidelberg de origen clínico (2008) y otro ambiental (2011) concuerdan con el tamaño de un plásmido que codificaría para funciones de movilidad plasmídica en $S$. Heidelberg ${ }^{2,30,40}$. En nuestro estudio, ocho aislados mostraron plásmidos de $30 \mathrm{~kb}$ y uno de estos fue positivo para AmpC y el gen bla ${ }_{\text {CMY-2' }}$ coincidente con plásmidos de tamaño similar descritos en $S$. Heidelberg ${ }^{37,40}$. Aunque debemos considerar que los plásmidos, a pesar de coincidir en tamaño, no significa que sean idénticos. También en $S$. Heidelberg se han descrito plásmidos de 40 y hasta $150 \mathrm{~kb}^{31,34,40}$, incluyendo algunos que codifican para $\beta$-lactamasas tipo $\mathrm{AmpC}^{37}$, sin embargo, en nuestro estudio no descartamos la presencia de plásmidos de tamaños mayores en $S$. Heidelberg, debido a las limitantes de las técnicas utilizadas. Todo esto concuerda con antecedentes que indicarían que $S$. Heidelberg contiene un genoma variable, de diferentes tamaños, por la presencia de fagos y plásmidos ${ }^{2,12,30}$.

Un estudio de cepas de $S$. Heidelberg aisladas entre 1985 y 2011 en Estados Unidos de Norteamérica mostró que los genes de factores de virulencia en este serovar estaban altamente conservados ${ }^{2}$, coincidiendo con nuestros resultados, donde los genes de fimbrias (agfA, lpfA, stkD), el gen de invasión celular (invA) y sifA, gen que codifica 
para un factor de multiplicación bacteriana en macrófago, se detectaron en todos los aislados. Contrariamente, cepas de $S$. Heidelberg en Brasil mostraron variabilidad en la presencia de $a g f A^{6}$ y otro estudio en Reino Unido mostró variación en $s t k \mathrm{D}^{15}$. Además, corroboramos que el gen fimbrial pefA descrito en algunos serovares de Salmonella ${ }^{41}$ no está en $S$. Heidelberg, coincidiendo con otro estudio anterior ${ }^{2}$.

El antibiotipo y las características genéticas de S. Heidelberg aisladas en Chile entre 2006 y 2011, mostró que este serovar presenta alta susceptibilidad a antimicrobianos usados de primera línea en el tratamiento de salmonelosis, con excepción de tetraciclina y ciprofloxacino, donde encontramos resistencia y susceptibilidad disminuida. Además, este estudio nos alerta sobre la circulación de clones que presentan $\beta$-lactamasas tipo BLEE y AmpC plasmídicas en nuestro país, lo que puede generar falla de tratamiento en pacientes con infecciones por este serovar $y$, también, da cuenta de la posibilidad que tienen estas cepas de diseminar la resistencia a cefalosporinas de tercera generación a otras enterobacterias. S. Heidelberg aisladas en Chile tendrían características similares a cepas de otras partes del mundo, codificando para genes de virulencia y con plásmidos de tamaño y número variable. En base a este estudio, proponemos que la presencia de plásmidos en $S$. Heidelberg podría utilizarse junto a otras herramientas de subtipificación bacteriana. Por otra parte, las características estudiadas no explicarían el aumento de casos ocurridos en Chile por S. Heidelberg el año 2011 respecto a años anteriores.

Agradecimientos: Al Instituto de Salud Pública de Chile por la donación de las cepas de $S$. Heidelberg utilizadas en este estudio. Ana Zepeda Ortega, Tecnólogo Médico y PhD. Escuela de Tecnología Médica Universidad de Valparaíso por su colaboración en el análisis estadístico.

\section{Referencias}

1. Andrysiak AK, Olson AB, Tracz D, Dore K, Irwin R, Ng LK, et al. Genetic characterization of clinical and agrifood isolates of multi drug resistant Salmonella enterica serovar Heidelberg from Canada. BMC Microbiol 2008; 8: 89.

2. Hoffmann M, Zhao S, Pettengill J, Luo Y, Monday SR,
Abbott J, et al. Comparative genomic analysis and virulence differences in closely related salmonella enterica serotype Heidelberg isolates from humans, retail meats, and animals. Genome Biol Evol 2014; 6 (5): 1046-68.

3. Green A, Defibaugh-Chavez S, Douris A, Vetter D, Atkinson R, Kissler B, et al. Intensified Sampling in Response to a Salmonella Heidelberg outbreak associated with multiple establishments within a single Poultry corporation. Foodborne Pathog Dis 2018; 15 (3): 15360 .

4. Zhao S, White DG, Friedman SL, Glenn A, Blickenstaff $\mathrm{K}$, Ayers SL, et al. Antimicrobial resistance in Salmonella enterica serovar Heidelberg isolates from retail meats, including poultry, from 2002 to 2006. Appl Environ Microbiol 2008; 74 (21): 6656-62.

5. Otto S J, Carson CA, Finley RL, Thomas MK, Reid-Smith RJ, McEwen SA. Estimating the number of human cases of ceftiofur-resistant Salmonella enterica serovar Heidelberg in Quebec and Ontario, Canada. Clin Infect Dis 2014; 59 (9): 1281-90.

6. Borsoi A, Santin E, Santos LR, Salle CT, Moraes HL, Nascimento VP. Inoculation of newly hatched broiler chicks with two Brazilian isolates of Salmonella Heidelberg strains with different virulence gene profiles,antimicrobial resistance, and pulsed field gel electrophoresis patterns to intestinal changes evaluation. Poult Sci 2009; 88 (4): 750-8.

7. Chittick P, Sulka A, Tauxe V, Fry AM. A summary of national reports of foodborne outbreaks of Salmonella Heidelberg infections in the United States: clues for disease prevention. J Food Prot 2006; 69 (5): 1150-3.

8. Folster JP, Pecic G, Rickert R, Taylor J, Zhao S, Fedorka-Cray PJ, et al. Characterization of multidrug-resistant Salmonella enterica serovar heidelberg from a ground turkey-associated outbreak in the United States in 2011. Antimicrob Agents Chemother 2012; 56 (6): 3465-6.

9. Folster JP, Pecic G, Singh A, Duval B, Rickert R, Ayers S, et al. Characterization of extended-spectrum cephalosporin-resistant Salmonella enterica serovar Heidelberg isolated from food animals, retail meat, and humans in the United States 2009. Foodborne Pathog Dis 2012; 9 (7): 638-45.

10. Meunier D, Jouy E, Lazizzera C, Doublet B, Kobisch $\mathrm{M}$, Cloeckaert A, et al. Plasmid-borne florfenicol and ceftiofur resistance encoded by the floR and blaCMY-2 genes in Escherichia coli isolates from diseased cattle in France. J Med Microbiol 2010; 59: 467-71.

11. Aarestrup FM, Hasman H, Olsen I, Sorensen G. International spread of bla(CMY-2)-mediated cephalosporin resistance in a multiresistant Salmonella enterica serovar 
Heidelberg isolate stemming from the importation of a boar by Denmark from Canada. Antimicrob Agents Chemother 2004; 48 (5): 1916-7.

12. Zhang Y, LeJeune JT. Transduction of bla(CMY-2), tet(A), and tet(B) from Salmonella enterica subspecies enterica serovar Heidelberg to S. Typhimurium. Vet Microbiol 2008; 129 (3-4): 418-25.

13. Lindsey RL, Fedorka-Cray PJ, Frye JG, Meinersmann R $\mathrm{J}$. Inc A/C plasmids are prevalent in multidrug-resistant Salmonella enterica isolates. Appl Environ Microbiol 2009; N75 (7): 1908-15.

14. Cejas D, Vignoli R, Quinteros M, Marino R, Callejo R, Betancor L, et al. First detection of CMY-2 plasmid mediated beta-lactamase in Salmonella Heidelberg in South America. Rev Argent Microbiol 2014; 46 (1): 30-3.

15. Bronowski C, Winstanley C. Identification and distribution of accessory genome DNA sequences from an invasive African isolate of Salmonella Heidelberg. FEMS Microbiol Lett 2009; 298 (1): 29-36.

16. Fica A, Acosta G, Dabanch J, Perret C, Torres M, López $\mathrm{J}$, et al. [Salmonellosis outbreaks and the size and role of the Chilean State]. Rev Chilena Infectol 2012; 29: 207-14.

17. Silva J, Aravena C, Araya J, Colque-Navarro P, Kühne I, Mollby R. [Biochemical phenotypes and phage types of Salmonella enteriditis strains isolated in Antofagasta during the period 1997-2000]. Rev Med Chile 2003; 131 : 837-45.

18. Grimont P, Weill FX. Antigenic formulae of the Salmonella serovars, $7^{\text {th }}$ edition. WHO Collaborating Centre for Reference and Research on Salmonella. Institut Pasteur, Paris. France. 2007; p. 166.

19. Bauer AW, Kirby WM, Sherris JC, Turck M. Antibiotic susceptibility testing by a standardized single disk method. Am J Clin Pathol 1966; 45 (4): 493-6.

20. CLSI (Clinical and Laboratory Standards Institute). Performance Standards for antimicrobial susceptibility testing. Information Supplement M10-S23; Twenty-third edition. 2013. Wayne PA, USA.

21. Takahashi S, Nagano Y. Rapid Procedure for Isolation of Plasmid DNA and Application to Epidemiological Analysis. J Clin Microbiol 1984; 4: 608-13.

22. Fadl AA, Nguyen AV, Khan MI. Analysis of Salmonella enteritidis Isolates by Arbitrarily Primed PCR. J Clin Microbiol 1995; 33 (4): 987-9.

23. Collinson SK, Emody L, Trust TJ, Kay WW. Thin Aggregative fimbriae from diarrheagenic Escherichia coli. J Bacteriol 1992; 174 (13): 4490-5.

24. Zhao S, White DG, McDermott JL, Friedman SL, English L, Ayers S, et al. Identification and Expression of Cephamycinase blaCMY genes in Escherichia coli and
Salmonella Isolates from Food Animals and Ground Meat. Antimicrob Agents Chemother 2001; 45 (12): 3647-50.

25. Baümler AJ, Heffron F. Identification and sequence analyses of $l p f A B C D E$, a putative fimbrial operon of Salmonella Typhimurium. J Bacteriol 1995; 177 (8): 2087-97.

26. Haneda T, Okada N, Nagazawa N, Kawakami T, Danbara H. Complete DNA sequence and comparative analysis of the 50-kilobase virulence plasmid of Salmonella enterica serovar Cholerasuis. Infect Inmun 2001; 69 (4): 2612-20.

27. Skyberg JA, Logue CM, Nolan LK. Virulence genotyping of Salmonella spp. with multiplex PCR. Avian Dis 2006; 50 (1): 77-81.

28. Sanger F, Nicklen S, Coulson AR. DNA sequencing with chain-terminating inhibitors. Proc Natl Acad Sci USA 1977; 74: 5463-7.

29. Phornphisutthimas S, Thamchaipenet A, Panijpan B. Conjugation in Escherichia coli. Biochem Mol Biol Educ 2007; 35 (6): 440-5.

30. Fricke WF, Mammel MK, Dermott PF, Tartera C, White DG, Leclerc JE, et al. Comparative genomics of $28 \mathrm{Sal}$ monella enterica isolates: evidence for CRISPR-mediated adaptive sublineage evolution. J Bacteriol 2011; 193 (14): 3556-68.

31. Kaldhone P, Nayak R, Lynne AM, David DE, Mc Dermott PF, Logue CM, et al. Characterization of Salmonella enterica serovar Heidelberg from turkey-associated sources. Appl Environ Microbiol 2008; 74 (16): 503846.

32. Ibar MP, Vigo G, Piñeyro P, Caffer MI, Quiroga P, Perfumo C, et al. Serovariedades de Salmonella enterica subespecie enterica en porcinos de faena y su resistencia a los antimicrobianos. Rev Argen Microbiol 2009; 41 (3): 156-62.

33. Junod T, López-Martin J, Gädicke P. Estudio de susceptibilidad antimicrobiana de Salmonella enterica en muestras de origen animal y alimentario. Rev Med Chile 2013; 141 (3): 298-4.

34. Han J, David DE, Deck J, Lynne AM, Kaldhone P, Nayak, et al. Comparison of Salmonella enterica serovar Heidelberg isolates from human patients with those from animal and food sources. J Clin Microbiol 2011; 49 (3): 1130-3.

35. Kim SY, Lee SK, Park MS, Na HT. Analysis of the Fluoroquinolone Antibiotic Resistance Mechanism of Salmonella enterica Isolates. J Microbiol Biotechnol 2016; 26 (9): 1605-12.

36. El-Tayeb MA, Ibrahim ASS, Al-Salamah AA, Almaary KS, Elbadawi YB. Prevalence, serotyping and antimi- 
crobials resistance mechanism of Salmonella enterica isolated from clinical and environmental samples in Saudi Arabia. Braz J Microbiol 2017; 48: 499-508.

37. Dorr PM, Tadesse DA, Zewde BP, Fry P, Thakur S, Gebreyes WA. Longitudinal Study of Salmonella Dispersion and the Role of Environmental Contamination in Commercial Swine Production Systems. Appl Environ Microbiol 2009; 75 (6): 1478-86.

38. Keelara S, Thakur S. Dissemination of plasmid-encoded AmpC beta-lactamases in antimicrobial resistant Salmonella serotypes originating from humans, pigs and the swine environment. Vet Microbiol 2014; 173

$$
\text { (1-2): 76-83. }
$$

39. Gieraltowski L, Higa J, Peralta V, Green A, Schwensohn C, et al. National Outbreak of Multidrug Resistant Salmonella Heidelberg Infections Linked to a Single Poultry Company. PLoS One 2016; 11 (9): e0162369.

40. Han J, Lynne AM, David DE, Tang H, Xu J, Nayak R, et al. DNA sequence analysis of plasmids from multidrug resistant Salmonella enterica serotype Heidelberg isolates. PLoS One 2012; 7 (12): e51160.

41. van Asten AJ, van Dijk JE. Distribution of "classic" virulence factors among Salmonella spp. FEMS Immunol Med Microbiol 2005; 44 (3): 251-9. 